\title{
Biotechnology in a snapshot
}

\author{
Aris Persidis
}

W ho would have thought that at the advent of the $21^{\text {st }}$ century, we would have witnessed the mapping of the entire human genome, the cloning of more than one animal, new drugs aimed at chain reactions deep inside cells, several artificial tissues and organs at various stages of development, use of natural language principles to analyze biological information, and all the other dazzling wonders of modern biotechnology? For the past 3 years, I have been discussing these wonders in the pages of the Industry Trends column of Nature Biotechnology.

The concept for the column sounds deceptively simple: in about 1,500 words, provide a concise account of the historical evolution, current state, challenges, and possible future directions of a biotechnology field for an audience of participants and observers, of scientists and business folks. The articles have a consistent format across the topics covered, such that they ultimately provide concise snapshots of biotechnology areas that are all within the same framework, allowing reasonable comparisons.

On occasion, I have co-authored these articles with highly respected colleagues whose unique expertise helped add significant twists to the themes, and I am grateful to them for their contributions. I would like to thank professor Atul Nerkar at the Columbia Business School (New York) for his contributions and advice. I am also indebted to Andreas Persidis and John Strouboulis at Biovista, who provided all the critical support in terms of data mining and sharing of their databases on technology status and business development updates. And of course, I am grateful to the staff of Nature Biotechnology past and present for support and editorial direction.

\section{Hard choices}

As in all compilations, some hard choices in selecting content had to be made. I have strived to balance well-recognized areas with recently emerging areas that promise exciting possibilities for the future. Obviously, no selection can be perfect, but the coverage here does include some of the hottest areas and issues in biotechnology

Aris Persidis is managing director of RheoGene, 706 Forest Street, Charlottesville, VA22903 (apersidis@rheogene.com). today. For those areas and participants who are excluded from the current collection, there will be opportunities for coverage in the future.

The content of this compilation focuses mainly on technology platforms and disease areas, but I have also included two articles on business issues. An entirely new article on corporate intelligence systems (pp. 5-6) is included because this field will be increasingly important to cope with the deluge of data and information arising from biotechnology research (much of the data and information for the articles herein, for example, was compiled using a systematic corporate intelligence system).

Of course, having "actionable information" isn't useful unless one can lever-

age it for business competitiveness. Who better to talk about this than Stelios Papadopoulos (pp. 3-4), who has kindly contributed a few thoughts on how aggressive funding coupled with top science, people, and execution are critical components of future successful biotechnology companies.

On the disease front, I have included some of the biggest killers and debilitators. According to my estimation, the total disease burden-direct and indirect-of the eight diseases covered in these pages is a staggering $\$ 500$ billion. Nevertheless, all of these diseases are manageable today; certainly not perfectly, but with education, prevention, and aggressive treatment, they seem less fearsome, which is a credit to the industry and to the advances described throughout this collection and elsewhere.

\section{Technology platforms}

In the final section of the supplement, several articles cover many of the technology platforms offered by biotechnology companies plus some less familiar ones, such as data mining (pp. 35-36) and xenotransplantation ( $p p$ 53-55). Data mining is a field that is constantly redefining itself, capitalizing on numerous information technology advances to re-define how biological information is collected, stored, analyzed and transmitted, and is an area given increasing attention from major players. Xenotransplantation is benefiting from major advances in immunomodulation and organ engineering and promises to address critical organ shortages. Organ engineering itself is covered in another article on tissue engineering (pp. 56-58), where there have been some spectacular recent advances due to the intersection of new materials, electronics, and biology.

I have also covered biochips (pp. 43-44), bioinformatics (pp. 31-34), and proteomics (pp. 45-46)-topics that have proved some of the most popular over the years. In all of these areas, the hardware and software is advancing at breathtaking speed, and crossover of the technology is significant. Thus, a proteomics experiment often includes a gene or protein array, and bioinformatics tools are used to analyze the data. Advances in automation and sensitivity make this combined platform very successful, as demonstrated by the high degree of corporate interest.

\section{Keeping pace}

Feeding these technology platforms are fields of biology where research is rapidly advancing. For example, progress in our understanding of the signal transduction cascades (pp. 37-39) from the cell surface to the nucleus, and all points in between, is not only driving a very robust reagent industry, but also opening up new areas of drug discovery that connect disease phenotypes with precise events inside cells. It is also facilitating our understanding of how drugs precisely affect the whole physiology of a cell, and not just the molecule they are designed to interact with. Thus, we can expect a new generation of drugs with reduced side effects and improved specificity, which is a major driver of the biotechnology and pharmaceutical industry at present.

As biotechnology moves inexorably forward, we shouldn't simply stand in awe. We should be as informed as we can, so that we understand better and appreciate the advances, issues, and problems for what they are. Hopefully, this collection will help in a small way toward that goal. 